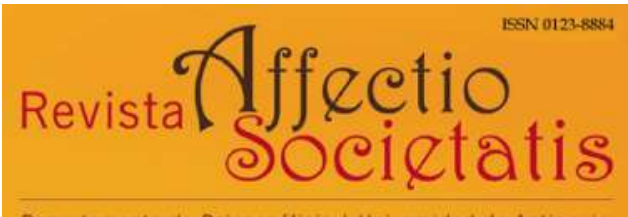

Departamento de Psicoanálisis / Universidad de Antioquia

Revista Affectio Societatis

Departamento de Psicoanálisis

Universidad de Antioquia

revistaaffectiosocietatis@udea.edu.co

ISSN (versión electrónica): 0123-8884

Colombia

2020

María del Mar Pérez Arizabaleta, Johnny Javier Orejuela Gómez

Sujeto, síntoma, dispositivo y terapeuta: Una mirada hacia la responsabilidad subjetiva en el campo de la salud mental

Revista Affectio Societatis, Vol. 17, N. ${ }^{\circ} 32$, enero-junio de 2020

Art. \# 9 (pp.191-213)

Departamento de Psicoanálisis, Universidad de Antioquia

Medellín, Colombia 


\title{
SUJETO, SÍNTOMA, DISPOSITIVO Y TERAPEUTA: UNA MIRADA HACIA LA RESPONSABILIDAD SUBJETIVA EN EL CAMPO DE LA SALUD MENTAL
}

María Del Mar Pérez Arizabaleta ${ }^{1}$

Universidad Antonio Nariño mariadelmarperezari@outlook.com ORCID: 0000-0001-8537-6696

\author{
Johnny Orejuela² \\ Universidad Eafit \\ jorejue2@eafit.edu.co \\ ORCID: 0000-0001-9181-463X \\ DOI: 10.17533/udea.affs.v17n32a09
}

\section{Resumen}

En la actualidad, la salud mental es un tema que genera preocupación. El incremento de personas diagnosticadas con trastornos mentales causa alarma a nivel mundial. La hospitalización y medicalización son "soluciones" puestas en diálogo y tensión con visiones que apuntan por tratamientos donde el sujeto construya un saber-hacer con su síntoma. En este último punto es en el que se enfoca el presente artículo: discutir la responsabilidad subjetiva que tiene el sujeto dentro del tratamiento. Para lo anterior, se propone llevar a cabo la discusión de la responsabilidad subjetiva por medio de la disertación alrededor de cuatro ejes de análisis: la

1 Psicóloga, especialista en Psicología Clínica con Orientación Psicoanalítica. Candidata a magíster en Psicología con énfasis en investigación. Joven investigadora del grupo de investigación en Psicología (GRIPSI) de la Universidad Antonio Nariño.

2 Doctor en Psicología, Universidad de São Paulo. Especialista en Psicología Clínica con Orientación Psicoanalítica. Profesor Titular y Jefe del Departamento de Psicología, Universidad Eafit. 
concepción de sujeto y de síntoma, el dispositivo de tratamiento y la posición del terapeuta.
Palabras clave: Responsabilidad subjetiva, salud mental, sujeto, síntoma y dispositivo.

\section{SUBJECT, SYMPTOM, DEVICE, AND THERAPIST: A LOOK AT SUBJECTIVE RESPONSIBILITY IN THE FIELD OF MENTAL HEALTH}

\begin{abstract}
Today, mental health is an issue of concern. The increase in people diagnosed with mental disorders is causing alarm worldwide. Hospitalization and medicalization are "solutions" put in dialogue and tension with visions that aim at treatments where the subjects build a know-how with their symptom. This paper focuses on this last point: discussing the subjective responsibility that the subject has
\end{abstract}

within the treatment. For this purpose, it is proposed to carry out the discussion of the subjective responsibility through the dissertation around four axes of analysis: the conception of subject and symptom, the treatment device, and the therapist's position.

Keywords: Subjective responsibility, mental health, subject, symptom, device.

\section{SUJET, SYMPTÔME, DISPOSITIF ET THÉRAPEUTE : UNE APPROCHE SUR LA RESPONSABILITÉ SUBJECTIVE DANS LE DOMAINE DE LA SANTÉ MENTALE}

\section{Résumé}

La santé mentale est aujourd'hui un sujet qui préoccupe. L'augmentation de personnes atteintes de troubles mentaux alarme les autorités au niveau mondial. L'hospitalisation et la médicalisation apparaissent comme des «solutions» qui doivent être confrontées à des approches visant des traitements où le sujet construit un savoir-faire avec son symptôme. C'est précisément sur ce point que se focalise cet article : examiner la responsabilité subjective du sujet dans son traitement. Pour ce faire, l'on propose de réaliser la discussion sur la responsabilité subjective autour 
de quatre axes d'analyse : la concep- Mots-clés : responsabilité subjective, tion de sujet, celle de symptôme, le santé mentale, sujet, symptôme et dispositif de traitement et la position dispositif. du thérapeute.

Recibido: 13/04/2019 • Aprobado: 20/10/2019 
A partir del siglo XIX -época en la que se le confiere el cuidado de los "locos" a la medicina y, por consiguiente, la salud mental se convierte en foco de investigaciones y reflexiones-, la búsqueda de causas y soluciones al sufrimiento y a la patología mental ha desembocado en la aplicación de tratamientos que buscan la eficacia en términos de inmediatez y del menor dolor posible en aquel camino hacia alguna solución. Orientada por la efectividad terapéutica, la ciencia médica ha hecho de la salud mental: una especialidad que se enfoca en el cuerpo como instrumento y que intenta equilibrar en él, a través de medicamentos, aquello que se ha alterado.

Por su parte y como intervención alternativa -desde sus inicios y de acuerdo a su fundador- el psicoanálisis es considerado: una psicoterapia que se distancia, más no se contrapone, de la terapéutica médica por su visión de sujeto, síntoma y tratamiento. En relación a esto, Freud (1992a) aclaró: "no es posible comprender una psiquiatría profundizada en sentido científico sin un buen conocimiento de los procesos de la vida del alma que van por lo profundo, de los procesos inconscientes" (p. 245); así pues, -al visionar que la vía de la psiquiatría no era suficiente para el abordaje de los procesos psíquicos- Freud plantea al psicoanálisis como un método terapéutico que, sin prometer milagros, le apuesta a la cura. Dicho método centra su atención en el inconsciente y da valor a la palabra, al sentido; por lo que la libre asociación y la transferencia son fundamentales desde el marco del silencio del analista, se demanda del paciente: insistencia, trabajo y esfuerzos (temporales y económicos).

De acuerdo con lo anterior, se podría pensar en las influencias que el psicoanálisis ha tenido en referencia a la salud mental; aunque Freud no fue psiquiatra, sus desarrollos teóricos fueron compartidos en este ámbito como una posición que podía aportar a tratamientos médicos de la época y ponerles en vilo. Lo que nos permite afirmar que el psicoanálisis aplicado a la salud mental tuvo lugar desde la génesis de la corriente freudiana, implicando al sujeto analizante tanto en el malestar que le acontece como en el tratamiento que se le ofrece. Basta recordar con Lacan (2003a), el historial clínico de Dora, exactamente la intervención de Freud con la que la cuestiona desde su escucha activa: "mira, le dice, cuál es tu propia parte en el desorden 
del que te quejas" (Lacan citando a Freud, p. 208); una expresión que expone a la clínica de la salud mental, la implicación del sujeto en el tratamiento de su sufrimiento -alejándose, así, de intervenciones limitadas a cuestiones extrínsecas que dejan al sujeto en posición pasiva, simplemente, de paciente-.

No obstante, apostarle a la implicación subjetiva del paciente en su tratamiento es un tema que demanda un análisis detallado puesto que debe tenerse claridad sobre lo que es entendido como "implicación" y sobre lo que se debe tener en cuenta para analizar -si en un dispositivo clínico del campo Psi (psiquiatría, psicoanálisis y psicología) eso se posibilita-. Respecto a lo primero, al discutir sobre la implicación del sujeto en su tratamiento hacemos referencia a la propia responsabilidad que este tiene frente a su sufrimiento y su tratamiento -en otras palabras, la implicación del sujeto respecto a su malestar y, por qué no, de un "saber hacer" sobre su síntoma-. Dado que, preguntarse por la responsabilidad subjetiva en el campo de la salud mental requiere un abordaje desde indicadores específicos -indicadores indispensables en cada tratamiento-, este trabajo concibe los términos: dispositivo terapéutico, terapeuta, sujeto y síntoma como las categorías brújulas que ayudarán a desplegar el análisis al respecto. Vale aclarar que el interés por esta conceptualización parte de la hipótesis que considera que: en la medida en la que se le ofrece al paciente un dispositivo con el que la subjetividad sea escuchada y cuyo tratamiento se oriente a implicar al sujeto como agente activo (responsable en el sufrimiento que lo aqueja y, sobre todo, en su tratamiento, un "saber hacer" con su síntoma), es posible que disminuyan los episodios agudos de la enfermedad mental y, por ende, el reingreso de pacientes a urgencias y hospitalizaciones psiquiátricas.

\section{Un recorrido sobre la definición de la responsabilidad subjetiva}

Inicialmente, para plantear la definición de "responsabilidad subjetiva", se debe tener en cuenta que los grandes exponentes del psicoanálisis (Freud y Lacan) no desarrollaron teóricamente este concepto; el 
interés por su conceptualización surge hace aproximadamente veinte años en discusión con lo que se entendía en el marco jurídico. Al respecto, Eidelsztein (2015) describe que:

La responsabilidad subjetiva es aquella en la que se le atribuye la culpa al individuo que realiza la conducta en cuestión sin dolor ni intensión y que debe reparar el daño. Siempre se considera, en este tipo de responsabilidad jurídica, al sujeto en posición de causa, ya sea por omisión, negligencia, descuido, etc. (p. 3).

En esta medida, la expresión "responsabilidad subjetiva" guarda estrecha relación con el modo de concepción del hombre en la sociedad; en este caso, un hombre que -desde una postura filosófica liberal, burguesa e individualista- se refleja como: "libre y responsable que, debe hacerse cargo de los hechos de su vida, aunque los produce sin la voluntad de hacerlos e, incluso, sin saberlo" (Eidelsztein, 2015, p. 4). También, dicha expresión emerge del ideario de "sujeto pensante" -es decir, de la terminología cartesiana cogito ergo sum-; aquel sujeto que es -para el contexto moderno occidental- digno de autorreflexión, autonomía y autofundación. En este orden de ideas y de acuerdo a Eidelsztein (2015), la "responsabilidad subjetiva" hace referencia al deber moral del sujeto de "hacerse cargo de todo aquello de lo cual se padece, goza, piensa, desea o actúa y, especialmente, se queja" (Eidelsztein, 2015, p. 11); teniendo como horizonte la idea social "Hazte cargo" [de ti mismo] -slogan que obedece a la garantía del discurso jurídico que ofrece respaldo de la palabra sin contrato, por el que: "todo acto y toda palabra tendrá en última instancia un individuo que dé garantía por aquellos, aunque sean descriptivamente inconscientes" (Eidelsztein, 2015, p. 6) -.

Respecto a lo que al psicoanálisis se refiere, el concepto de "responsabilidad subjetiva" se genera en un diálogo con el derecho, partiendo de la idea de "sujeto de la ciencia". Por lo tanto, desde esta óptica debe ser leída la célebre frase de Lacan (2003/1953): “De nuestra posición de sujeto somos siempre responsables" (p. 837). Ante ella, nos dice Eidelsztein (2015): "lo que Lacan propone es que debemos en psicoanálisis hacer un planteo claro y explícito sobre cuál es el concepto de sujeto con el que operamos y responder los psicoanalistas 
por ello" (p. 13); la intensión de Lacan es la de localizar el concepto de "sujeto" desde el cual opera el psicoanálisis. Además, Eidelsztein (2015) agrega:

No dice que cada uno de nosotros [los psicoanalistas $]^{3}$ debe hacerse responsable de sus asuntos de sujeto, sino que los analistas deben ser responsables, o sea, como ya vimos, deben poder responder por cómo plantean, postulan al sujeto en su teoría y en su práctica y en su diálogo con las ciencias y disciplinas afines. (p. 13).

Así pues, en la Modernidad, discutir sobre "responsabilidad subjetiva" llevaba a cuestionarse sobre la responsabilidad que corresponde al sujeto respecto a su tratamiento -psicoanalítico, médico, psicológico, psiquiátrico, etc. -, incluso reflexionar sobre la acción del terapeuta y su noción de "sujeto" y "síntoma". Sobre la posición de sujeto, Greiser (2012a) plantea que "siendo el goce el campo sobre el cual opera el psicoanálisis, la responsabilidad en psicoanálisis esta especificada por la relación que el sujeto tiene con el goce implicado en sus actos" (p. 73); por lo tanto -y de acuerdo a Lacan (2012)-, quien se hace responsable de su manera de gozar es a quien se le puede denominar como "persona adulta", de lo contrario se está en presencia de "niños generalizados" en tanto no se hacen responsables de sus modos de gozar ni de las consecuencias que esto conlleva.

Por lo tanto y en concordancia con las categorías que se han planteado para desplegar bases para el análisis de la responsabilidad subjetiva en el contexto de la salud mental, se puede afirmar que discutir sobre la responsabilidad subjetiva se fundamenta en la posición de quien realiza una intervención o tratamiento. Es así como se entiende que la responsabilidad subjetiva es un concepto desde el cual se sustenta la orientación psicoanalítica y sus principios éticos. Pues esto reúne tanto al sujeto analizante como al terapeuta y todo lo que el dispositivo conlleva, entre eso: la trasferencia. En esta medida, la posición política del psicoanálisis demanda una escucha del sujeto que permite una enseñanza propia de cada caso. Frente a esto, el analista debe asumir un semblante de sujeto supuesto saber y una posición

3 Corchetes insertos por los autores. 
también desde la docta ignorancia, condición que le permitirá al paciente la toma de la palabra. Es decir que, el analista es objeto causa de deseo y, por ende, causa por la que el sujeto toma su palabra, se apropia de su decir. De acuerdo con Consenza (2018), el analista demanda al sujeto la toma de su palabra, lo que demarca una radical diferencia entre el psicoanálisis y el discurso psiquiátrico.

\section{La responsabilidad subjetiva y la salud mental}

El surgimiento del concepto "salud mental" no es tan antiguo como se pudiera llegar a pensar, tampoco nace propiamente de la medicina como se infiere. Conforme al recorrido histórico que realiza Foucault (2015) sobre la locura se puede afirmar que ésta y su manera de proceder obedecen a cuestiones de orden público y que su aparición en la medicina data alrededor del siglo XIX. Este recorrido histórico presenta que, desde el inicio, la locura ha sido asociada socialmente con "el mal" y las maneras de intervenirla han estado en el orden del encierro y del control; es decir, se ha limitado a tratamientos que no posibilitan una implicación del sujeto en su malestar y tratamiento y que, en cambio, permiten que el sujeto entregue su sufrimiento patológico a otro (el médico) con el fin de buscar el alivio en lugares como: leprosarios o psiquiátricos, sitios de beneficencia, entre otros.

Desde el siglo XII al XIV (época de la alta Edad media y fin de las Cruzadas), la lepra era un sinónimo de la locura; por lo tanto, los leprosarios fueron los sitios para albergar este mal social. Allí también, para el siglo $\mathrm{XV}$, se albergaron las enfermedades venéreas, pero dado a que su apariencia no era tan espeluznante como la de la misma lepra, este tipo de enfermedades no confirieron ningún aislamiento y los sujetos que las padecieron fueron dignos de tratamientos médicos. Por esto, las enfermedades venéreas no reemplazaron la lepra en tanto representación social del mal; sino que fue la locura, esta se instaló en la sociedad como un sinónimo de mal digno de exclusión.

Desde el siglo XV hasta el XVII (Renacimiento), la locura convivió y fue concebida por la sociedad de diferentes maneras. Los locos 
representaban aquello que la sociedad no aceptaba como meritorio de ser humano -es decir: la enfermedad, el mal, y también la sensibilidad, la ridiculez, la ignorancia, la sinrazón y la debilidad humana-. Durante estos siglos, los "locos" fueron alojados y mantenidos por el Estado y así, también, habitantes de la cotidianidad social; pero cuando su número incrementó se exiliaron de las grandes ciudades. Se puede observar que, hasta el momento, la locura co-existía con otros fenómenos al interior de la sociedad, y que, al interior de esta, la locura no era tomada como objeto de tratamiento.

Con la llegada del racionalismo en el siglo XVII, la locura se convierte en el principal enemigo de la verdad y, así pues, del desarrollo científico también. La sociedad ya no permitía convivir con pensamientos insensatos, por lo que, desde la mitad de este siglo, la locura ha sido ligada a internados en auspicios; sin embargo, la población meritoria de estos sitios excedía la capacidad de los mismos, eran los jueces los encargados de evaluar y otorgar benéficamente la internación a aquellos que en realidad lo merecían. En concordancia con Foucault (2015): "el pobre, el miserable, el hombre que no puede responder de su propia existencia, en el curso del siglo XVI se ha vuelto una figura que la Edad Media no habría reconocido" (p. 91); en consecuencia con el racionalismo, se asumía al "loco" como un sujeto que no podía responder por su propia existencia y requería de un tercero quien pudiera "asumir" la responsabilidad del alienado. Vemos con esto que el gesto del encierro está rodeado de significados políticos, sociales, religiosos, económicos y morales; sobre todo se destaca, sin querer, la idea de que uno no responde por sí mismo, sino que otro decide por uno (en suma, la asunción no calculada de evasión de las propias preguntas respecto de lo que tengo como responsabilidad por mí mismo, en tanto que el otro asume toda responsabilidad por mí idea que se mantiene hasta hoy y que aquí invitamos a combatir-).

Es de esta manera que sucede "el gran encierro" y con ello, nuevas maneras de abordar y tratar la locura; en donde "la locura cede entonces su lugar en los manicomios al médico y comienza a aparecer, por consiguiente, el concepto de salud mental" (Pérez, 2010, p. 11). Se pasa de la locura del campo del orden público -adaptación social- al campo de la salud; en otras palabras, es el tránsito de un 
problema social a un problema conferido a la medicina y, por defecto, a la ciencia.

Bajo el objetivo de adaptación social y de la demanda científica, desde el siglo XIX la psiquiatría hace de los "locos" un área de investigación con fines de intervención en el que la configuración de la enfermedad se relaciona con una localización del mal en el cuerpo, es decir, se relaciona con una anatomía patológica (Braunstein, 2010); el enfoque nosológico de la psiquiatría plantea que en la enfermedad existen síntomas y signos observables, y “acepta, además, que los síntomas y signos indican un diagnóstico, el cual, a su vez, determina un pronóstico y un tratamiento" (Cooper, 1976, p. 14). Es así como el modelo médico, basado en la biología, da cuenta de procesos "objetivos" como soportes para la clasificación y tratamiento de problemas mentales (Braunstein, 2010), concibiendo al sujeto como un organismo biológico y dejando por fuera el estudio del sujeto, su síntoma y su singularidad. Lo que causa el surgimiento de posicionamientos que evidencian movimientos que debaten y cuestionan a la psiquiatría y, también, el despliegue de una vertiente psicoanalítica que se interesa por comprender y abordar el fenómeno de la salud mental, tomando en cuenta no tanto al cuerpo como sí a la subjetividad.

Desde el psicoanálisis, Miller (2010) discute que la definición de salud mental, hoy en día, no difiere del orden público pues el criterio para designar la perturbación mental está dado por la capacidad de comportarse en sociedad, de respetar las normas, es decir, de estar alineados al discurso del amo contemporáneo; un amo que adapta y, con ello, acalla los síntomas y desdibuja la singularidad. Desde una perspectiva lacaniana, estas cuestiones no obedecen en absoluto a una posibilidad terapéutica del psicoanálisis, pues si nos regimos bajo el concepto de "adaptación" se

negaría algo esencial del descubrimiento freudiano, es decir, el hecho de que en el ser humano habita algo radicalmente incurable ${ }^{4}$ (...) y si el psicoanálisis ha de servir para algo, para alguien, tendrá

4 Y esta dimensión de lo incurable denota una posición ante lo real. 
que contar con ese real como condición esencial de su acto. (Pérez, 2010, p. 16).

Entonces, el psicoanálisis no tiene como objetivo ofrecer sujetos adaptados, su tarea es "permitirle al sujeto asumir una posición para enfrentar la existencia" (Pérez, 2010, p. 16); el psicoanálisis difiere de aquella manera de actuar frente a la salud mental que se sitúa en los límites del orden público y la obediencia al discurso del amo representado en el discurso médico psiquiátrico, es por esta razón específica -por la obediencia al discurso del amo- que Laurent (2000) plantea lo siguiente:

La salud mental existe, pero tiene poco que ver con lo mental, y muy poco que ver con la salud. Tiene relación con el Otro, y con el silencio. La salud mental es lo que asegura el silencio del Otro, así como la salud es el silencio de los órganos. (p. 135).

Es en esta medida que "la terapéutica psicoanalítica se ha distanciado de la médica porque esta última se ha protocolizado y el protocolo impide la clínica" (Greiser, 2012b, p. 47); por lo anterior, el psicoanálisis posibilita al sujeto hacerse cargo de sí mismo, ubicándolo en oposición de un "para todos igual", pues el dispositivo analítico corresponderá al trabajo de la escucha del sujeto con su síntoma. Hemos de notar aquí, entonces, que se trata claramente de dos lógicas que se contraponen: la de la clínica médica [aditiva] y la de la clínica psicoanalítica [de la sustracción] (Quintero, 2017).

\section{Método de tratamiento, terapeuta, sujeto y síntoma}

Con el fin de desarrollar el tema central de este artículo, el de la responsabilidad subjetiva del sujeto en su tratamiento y malestar, a continuación se expone el paralelo entre dos maneras de intervenir en el campo de la salud mental: el discurso médico y el discurso psicoanalítico; como se ha comentado a lo largo de este artículo, la discusión entre estos discursos gira alrededor del método de tratamiento, la posición del terapeuta, el sujeto y el síntoma. Para ejemplificar la manera de intervención del discurso médico y la del psicoanalítico se expondrá una viñeta que ilustra los cuatro puntos en mención. 
Es necesario aclarar que dentro de lo que denominamos "discurso médico" se enmarca una amplia gama de especialidades; por lo que respecta a este texto, se discutirá de manera particular: la psiquiatría.

\section{Viñeta clínica}

A un servicio de urgencias de un hospital llega una joven, a la que llamaremos Verónica, con secuelas de autolesión. Su madre -quien es su acompañante- afirma que la ha encontrado en el baño, lacerándose los antebrazos; aunque esto es visible a los ojos, Verónica lo niega, refiriendo que se encuentra muy bien.

El médico realiza el ingreso y en primera instancia indaga datos personales y antecedentes de la paciente. Esta entrevista demuestra que Verónica es una joven tranquila, carismática y responsable; incluso, que vive con su madre y una hermana mayor por pocos años, ambas trabajan todo el día, razón por la que Verónica evita estar en casa y busca compañía en sus amigos durante el día. En este ingreso al servicio también se indaga por los antecedentes familiares de enfermedad mental, antecedentes médicos y quirúrgicos; pero no se encuentra nada relevante.

Posteriormente, se hace una revisión física y examen mental en los que se observa: un leve temblor en las manos, labilidad afectiva, tono de voz bajo, tartamudez, en ocasiones se presenta bloqueo en el pensamiento -sin ideas delirantes ni sensaciones alucinatorias-, distorsión del recuerdo, confabulación, cuando se le pregunta por el suceso ocurrido (laceración en los brazos). En definitiva: orientada en tiempo, lugar y persona; juicio debilitado.

Seguido a lo anterior, Verónica ingresa a hospitalización bajo el pseudónimo de "paciente" y permanece allí durante quince días. Después egresa con prescripción médica de una sertralina diaria, dado que la autolesión -asumida por el médico y la familia como intento suicida- se encuentra relacionada con un diagnóstico de depresión; también se agregan remisiones a trabajo social y a un centro de rehabilitación, pues durante los días de hospitalización Verónica mencionó haber consumido sacol y clonazepam sin prescripción médica en 
varias ocasiones, suponiendo así: una problemática de adicción a las sustancias psicoactivas. Finalmente, también, se ordena control por psiquiatría cada tres meses.

\section{Discurso médico-psiquiátrico}

En 1905, Freud expuso la psicoterapia médica bajo la metáfora de la técnica de la pintura; en la pintura, el artista "sobre la tela en blanco deposita acumulaciones de colores donde antes no estaban" (1992e, p. 250). Relacionando esto con el discurso médico, se puede decir que para la época freudiana, a partir de la medicina se ofrecía un método de tratamiento basado en la adición, tanto en el lienzo como en el sujeto que acude a un tratamiento psiquiátrico se adicionan elementos. En la particularidad del sujeto se hace caso omiso del origen, fuerza y significación de los síntomas; por lo tanto, el médico -a través de ideas preconcebidas- diagnostica y percibe con el fin de lograr un tratamiento, es decir, una cura, una obra de arte. Tal y como lo vimos en el caso de Verónica, el método psiquiátrico se caracteriza por la adición en el tratamiento, allí los fármacos y remisiones son elementos fundamentales por añadir para lograr el bienestar.

En la época freudiana, las neurosis histéricas eran intervenidas medicamente por medio de la sugestión hipnótica; bajo esta técnica "el hipnotizador afirma encontrarse en posesión de un poder misterioso que arrebata al sujeto su voluntad" (Freud, 1992d, p. 119), la influencia de sus palabras es aquello que hace modificar los estados afectivos $y$, con ello, los malestares (Roudinesco, 2005). No obstante, varios casos demostraron que el éxito de la cura por este medio era limitado, puesto que no todos los pacientes respondían a la hipnosis sin interrupción de la resistencia y, además, se dio cuenta que en la disolución del vínculo médico/paciente los síntomas retornaban. Por esto, la sugestión hipnótica tiene como pilar al médico, siendo aquel el que proporciona la cura en la medida en que se identifica y modifica el malestar. Partiendo del hecho de considerar que el poder de la cura no debía estar únicamente en manos del médico sino también del sujeto que acude a él, Freud decide abandonar la sugestión hipnótica como técnica; aunque de esta rescata el poder de la palabra e identifica la resistencia al tratamiento como el primer obstáculo de la cura (Freud, 1992c). 
Al desistir de dicho método, Freud piensa en el método catártico como nueva vía para la cura. Por medio de este se le permitía al paciente recordar una vivencia traumática y así, liberar su carga afectiva para recuperar el principio de constancia; por lo tanto, "recordar y abreaccionar eran en aquel tiempo las metas que se procuraba alcanzar con auxilio del estado hipnótico" (Freud, 1991b, p. 149). En 1900, tomando del método catártico: la importancia de la descarga por medio de la palabra, Freud plantea el método de la asociación libre como regla fundamental del psicoanálisis; con este método desaloja al médico del poder que le daba la sugestión y le permite al paciente involucrarse en el trabajo de la cura; entonces, "la abreacción era relegada y parecía sustituida por el gasto de trabajo que el analizado tenía que prestar al vencer, como le era prescrito (por la obediencia a la regla psicoanalítica fundamental), la crítica a sus ocurrencias" (Freud, 1991b, p. 149).

De lo anterior es pertinente mencionar que, si bien la palabra estuvo presente en el camino metodológico del psicoanálisis, su puesta en escena no fue igual en todos los casos. En cada método el paciente expresaba su malestar a través de la palabra; sin embargo, el hacer con la palabra tuvo transformaciones: pasó de palabras "impuestas" (sugestión), a palabras que daban cuenta de recuerdos de vivencias con alta carga afectiva (método catártico), a palabras que daban cuenta de recuerdo de vivencias reprimidas con los que se ponía en juego el inconsciente y acaecía una batalla contra la resistencia (asociación libre).

En lo anterior se puede identificar el camino por el cual el psicoanálisis se distancia del discurso médico, basado en el tratamiento mediado por la adición; un camino que se emprende desde el método de la asociación libre y que concibe al síntoma, al sujeto y al terapeuta de manera diferente; una transición que pasa del saber expuesto y concentrado en el terapeuta al saber condensado en el sujeto y sólo supuesto en el terapeuta, que se traslada desde la lógica de la adición a la de la sustracción.

De acuerdo a la metáfora de la pintura planteada por Freud y a la viñeta clínica, se puede identificar que el sujeto desde el discurso médico es tomado como objeto de estudio y que es el médico y/o los acompañantes del paciente quienes tienen la voz y decisión sobre su devenir, 
llegando al punto de omitir lo que el paciente tiene por decir frente a su propia situación; en el caso de Verónica, es su madre y sus laceraciones en el cuerpo lo que plantean el motivo de consulta, es decir que el inicio y base de su tratamiento se dictamina desde lo evidenciable, y por esto el producto final se limitará a un tratamiento repetido -receta médica y remisiones preconcebidas- que se haya afuera de la implicación del sujeto como tal en el sufrimiento que le atañe, afuera de la posibilidad de que sea la misma paciente quien cree una invención para aquello que le aqueja. Por todo lo anterior se puede afirmar que desde el discurso médico el sujeto queda despojado tanto de su cuerpo y de su malestar como de lo que él mismo puede llegar a saber-hacer para sentirse mejor (Clavreul, 1983); aquí el que sabe es el médico.

La posición del terapeuta, en este caso el médico, en este tipo de tratamientos se ubica desde el campo del saber, operando bajo ideas preconcebidas. Ha de saberse que para el siglo XIX, la sociedad y la ciencia habían confiado a la medicina: la investigación y definición de formas anormales de vivir (Braunstein, 2010), llevando a articular los males en el cuerpo y a clasificarlos por medio de criterios de anormalidad. Por esto, el tratamiento médico se encarga del cuerpo, chequeando el buen funcionamiento fisiológico mediante el seguimiento de signos corporales; esto se puede observar en el examen físico y mental de la viñeta clínica, procedimiento que realiza un psiquiatra al ingreso de un paciente a una unidad de salud mental. La descripción cualitativa o cuantitativa de la conducta motora, el afecto, el pensamiento, la senso-percepción, la conciencia, la orientación, la inteligencia, el juicio y el raciocinio son las guías para levantar criterios diagnósticos y plantear, así, un tratamiento que no implique al sujeto y perpetúe su goce en el síntoma.

Sobre lo anterior, Cooper (1976) esboza alrededor de la psiquiatría que "el enfoque nosológico postula que al tratarse de una enfermedad, hay síntomas y signos observables en una persona (...) Se acepta, además, que los síntomas y signos indican un diagnóstico, el cual, a su vez, determina un pronóstico y un tratamiento" (pp. 13-14); de modo que, "el conocimiento de las enfermedades es la brújula del médico y el éxito de la curación depende de un exacto conocimiento de la realidad" (Foucault, 2001, p. 43). Es de esta manera que el dis- 
curso médico y, puede decirse, varias psicoterapias por fuera de las psicoanalíticas son inscritas por Miller (2001) en lo que Lacan nombra como "discurso del amo".

El síntoma en el discurso médico es abordado como un problema que requiere ser retirado, silenciado u ocultado; los síntomas son entendidos bajo el lente de la clasificación. En el discurso médico, el síntoma -aquello que desde el psicoanálisis es propio de cada sujeto- debe ser fisiológicamente ubicado, nosológicamente abordado y terapéuticamente eliminado de acuerdo con patrones de tratamientos estandarizados; la búsqueda de causas y localizaciones de la enfermedad subordina la vida misma del paciente -expresada por medio de las ocurrencias, los sueños, el chiste y demás formaciones del inconsciente-. Luego, y de acuerdo a lo que dice Freud (1992a): "la psiquiatría clínica hace muy poco caso de la forma de manifestación y del contenido del síntoma individual" (p. 235), expresión que expone un punto esencial del psicoanálisis.

Al centrar la intervención y el tratamiento en la nosología, la psiquiatría se distancia de una concepción de sujeto capaz de afrontar y buscar soluciones autónomas frente al malestar, de la construcción de un síntoma singular y, con esto, de un tratamiento del caso por caso; por lo que se aleja de la posibilidad de entablar una relación médico/ paciente que se distinga de la de observador/observado que sólo supone un dominio del médico sobre el cuerpo y la vida del paciente.

\section{Discurso psicoanalítico}

Contrario a la destreza médica, Freud metaforiza la psicoterapia psicoanalítica con la técnica de la escultura; en ella se "quita de la piedra todo lo que recubre las formas de la estatua contenida en ella" (1992/1901-1905, p. 249), la obra de arte toma la forma en la medida en que su inventor -el paciente- retira elementos. Es decir que, el producto de la psicoterapia psicoanalítica será, finalmente, una invención de autoría del paciente.

Con el fin de apartarse de la clínica de la adición -clínica del discurso médico-, Freud plantea el método de la asociación libre como 
una ruta hacia lo olvidado o caído bajo la defensa; con él constituye una clínica de la sustracción. Orientando sus intervenciones bajo este método y con el fin de distanciarse de movimientos que produjeren levantamientos de defensas que interfirieran en el tratamiento, la regla fundamental del espacio analítico se convirtió en invitar y permitir al paciente a decir todo lo que quisiera; por lo tanto, en el plano del psicoanálisis, por medio de la asociación libre, se practica el hablar de sí sin cohibiciones, evitando, en lo posible, que la consciencia moral interrumpa el habla fluida. Lo que denota un punto de ruptura frente a la viñeta planteada, en la que se observa que el sujeto fue anulado mediante saberes preconcebidos -diagnóstico y tratamiento que responden a los resultados de exámenes médicos-, no hubo cabida para la asociación libre y, con esto, ni siquiera para la escucha del sujeto.

En el plano de la técnica y de la relación analista/analizante se pone en juego, además del método de la asociación libre, la transferencia. En conformidad con Freud (1991b), para conquistar la cura psicoanalítica se necesita de la transferencia; quiere decir que es necesario que en el espacio analítico, el sujeto ponga en escena su inconsciente por medio de una serie o clísé de mociones de pulsión que han sido reprimidas. En consecuencia, se puede afirmar que el psicoanalista/terapeuta cumple la función de objeto sobre el que recaen las mociones y, además, que en un psicoanálisis el sujeto (analizante/ paciente) y su inconsciente son los que trabajan. Por medio del sujeto supuesto saber, la trasferencia se sostiene; es decir que, por medio de ese saber inconsciente -que supone el analizante sobre el psicoanalista- se construye la transferencia; aquí se logran efectos analíticos -quienes deciden ser analizantes y/o terapéuticos, o sea, ceder en el síntoma haciendo invenciones- .

Por lo anterior, en la psicoterapia psicoanalítica: el papel del sujeto y del médico cambia; ya no se encuentra un sujeto pasivo frente a su tratamiento y malestar, como lo veíamos en el discurso médico, sino que encontramos un paciente activo que a partir de su propio discurso -significante, inconsciente- se permite dar cuenta de su síntoma y de la búsqueda de su cura. Es importante resaltar que para ello "es preciso que el paciente cobre el coraje de ocupar su atención en los fenómenos de su enfermedad" (Freud, 1991/1911-1913, p. 154); 
es decir que el sujeto trabaje, encare y se haga cargo de su síntoma, si lo que desea es la cura.

Por su parte el psicoanalista ${ }^{5}$, con ayuda de la transferencia, tiene como función el poner en descubierto ante el analizante: las resistencias. En este proceso, el psicoanalista guía al sujeto hacia un rumbo en donde pueda darse cuenta por sí mismo que su actuación cotidiana hace parte de una repetición del pasado; el psicoanalista debe dar tiempo al sujeto para que re-elabore la resistencia y pueda vencerla; también, debe apartar sus ideas preconcebidas o prejuicios - fantasma- para entregarse al paciente por medio de "una atención parejamente flotante, a su propia actividad inconsciente, evitando en lo posible la reflexión y formación de expectativas conscientes" (Freud, 1992a, p. 235). Así pues, Freud afirma que "a estas enfermedades no las cura el medicamento sino el médico" (Freud, 1992a, p. 249).

De acuerdo a lo anterior, el psicoanálisis "plantea elevadas exigencias tanto al enfermo como al médico" (Freud, 1992e, p. 252); al paciente se le exige compromiso, trabajo, sinceridad, tiempo y dinero. Por ello, Freud no entiende al psicoanálisis como una psicoterapia digna de todo el mundo, puesto que emplearla o no va a depender y a justificarse acorde a cada caso. Empero, Freud mismo considera que el psicoanálisis puede aplicarse a otros campos del saber; por lo que existe la posibilidad de un psicoanálisis aplicado a la salud mental. En su Conferencia 16, titulada: "Psicoanálisis y Psiquiatría", Freud (1992a) ya mencionaba la analogía: "el psicoanálisis es a la psiquiatría lo que la histología a la anatomía" (p. 233); con ella motivaba a apartarse de la idea de incompatibilidad entre psicoanálisis y psiquiatría.

Por su parte, la concepción de síntoma en el psicoanálisis se escapa de la conceptualización nosológica del discurso médico. Aunque Freud entabló su conceptualización con base en la medicina -por vía de la neurosis obsesiva e histeria-, se puede afirmar que dicho término en el psicoanálisis ha sufrido transformaciones de la mano de los

5 Desde el psicoanálisis se toma distancia de aquella concepción de "médico", como lo denomina el discurso médico, ya que en el discurso psicoanalítico se le nombra como "psicoanalista". 
cambios metodológicos en el tratamiento (sugestivo, catártico, asociación libre). En la época del método catártico, el síntoma era entendido como el producto de una causa psíquica de un pasado remoto $y$, por lo tanto, el camino a la cura era el de llegar al recuerdo de la vivencia traumática que había sido reprimida de la consciencia por su alta carga afectiva; en esta visión, el síntoma era considerado como el conjunto de "símbolos mnémicos", es decir, símbolos del recuerdo sofocado que debían descargarse para lograr un principio de constancia (Freud, 1992c). De allí que el objetivo terapéutico consistía en recordar mediante de la hipnosis para descargar el afecto contenido.

Con el tiempo, la idea de síntoma descrita anteriormente cambió; en la Interpretación de los sueños, con el desarrollo teórico del "funcionamiento del proceso primario de la psiquis y la forma en que influye en la producción de nuestros pensamientos accesibles" (Freud, 1992c, p. 12), de la sexualidad infantil y del Complejo de Edipo se entendió el síntoma como: una formación del inconsciente. De este modo, el síntoma pone en disputa una tendencia inconsciente reprimida que aspira tanto a la satisfacción pulsional como al cumplimiento de un deseo inconsciente). Entonces, el síntoma ya no hace referencia a una vivencia traumática exterior, sino que en él se da cuenta de un sentido inconsciente -edípico- y de una satisfacción pulsional. El síntoma se convierte en la solución singular bajo la cual el sujeto vive, y por esto Freud propone que en una terapia psicoanalítica el síntoma no se debe silenciar -como se hace desde la terapia médica-, sino que se debe comprender su función en la economía psíquica. Frente a esto, nos dice:

No debemos olvidar que tampoco es posible situarnos ante la vida como fanáticos higienistas o terapeutas. Hemos de confesarnos que esta profilaxis ideal de las enfermedades neuróticas no puede ser beneficiosa para todos. Mucho de los que hoy se refugian en la enfermedad no resistirían el conflicto en las condiciones por nosotros supuestas; sucumbirían rápidamente o causarían algún grave daño, cosas ambas más nocivas que su propia enfermedad neurótica. (Freud, 1972, pp. 71-72).

En ese orden de ideas, el desarrollo de una psicoterapia psicoanalítica ocurre cuando se escucha al sujeto con ayuda del método de la asociación libre; sin embargo, en este proceso de indagación del sín- 
toma se presenta el obstáculo de la represión: "el analizado no recuerda, en general, nada de lo olvidado y reprimido, sino que lo actúa. No lo reproduce como recuerdo, sino como acción; lo repite, sin saber, desde luego, que lo hace" (Freud, 1991b, pp. 151-152). Por lo tanto -y a diferencia del discurso médico-, desde el psicoanálisis: el síntoma no obedece exclusivamente a una localización en el cuerpo sino que se expresa -a manera de repetición- en el actuar cotidiano. Respecto a lo anterior, Freud (1991b) suscita: "el analizado repite en vez de recordar, y repite bajo las condiciones de la resistencia" (p. 153); por lo que lo reprimido se expresa en forma de síntoma. En el acto analítico, dicha repetición no es un error y es indispensable y se pone en juego a manera de transferencia (Freud, 1991b).

Correspondiente a lo anterior, en 1973 Lacan se posicionó frente a la discusión del sentido del síntoma como apuesta del psicoanálisis y expuso que "el sentido es un efecto del significante, por lo tanto desplazó la definición de sujeto hacia el significante, separó el significante del sentido e invitó a aislar los significantes atrapados sin ningún sentido en el síntoma" (Miller, 2001, p. 8), pasando de la discusión sobre el sentido hacia el significante. Entonces, el síntoma se asume como una solución frente a un real que se impone; y de ese modo, Lacan (2001) asevera que: "el yo está estructurado exactamente como un síntoma. No es más que un síntoma privilegiado en el interior del sujeto" (p. 32), puesto que el yo actúa y se configura desde la solución (sintomática) que se ha autorizado.

A manera de conclusión, se podría decir que la discusión expuesta aquí permite dar a conocer que los métodos de tratamiento tradicionales en el campo de la salud mental tienden a des-responsabilizar al sujeto de su malestar y, por lo tanto, de su tratamiento. Sobre esto Goffman (2007) nos ofrece una metáfora que compara el hospital psiquiátrico con un taller de autos, siendo ambos lugares a los que se llevan cuerpos con el fin de recogerlos reparados después de un período pertinente en el que se "arreglan"; esta metáfora parece evidenciar: la posición asumida por los médicos en el campo de la salud mental y la concepción de sujeto y síntoma con las que guían sus intervenciones. Con todo ello podemos ratificar la propuesta de que la responsabilidad subjetiva puede ser un elemento crucial, el prisma, 
a partir del cual pueden revisarse o reposicionarse las dimensiones de análisis aquí propuestas, a saber: el dispositivo, la concepción de sujeto y síntoma y la posición del terapeuta, elementos que, en su conjunto, constituyen lo que en psicoanálisis se denominan "elementos estructurales del dispositivo clínico".

\section{Referencias bibliográficas}

Braunstein, N. (2010). Crítica de la clasificación internacional en psiquiatría. En N. Braunstein, Psiquiatría, teoría del sujeto, psicoanálisis (hacia Lacan) (pp. 11-28). México: Siglo veintiuno editores.

Clavreul, J. (1983/1978). El orden médico. Traducción de Martha Vasallo. En J. Clavreul, El orden médico (pp. 39-51). España: EMEGE, Industrias gráficas.

Consenza, D. (31 demayo de 2018). Efectos dela formacióny efectos desubjetivación en la enseñanza de la presentación de enfermos. En Revista Virtual Nueva Escuela Lacaniana (NEL), 10, Ciudad de México. Recuperado de http:// www.nel-mexico.org/index.php?sec=GLIFOS\&file=GLIFOS $/ 010 /$ Programa-de-presentacion/Efectos-de-formacion.html.

Cooper, D. (1976). Introducción. En Piatigorsky, K. (Trad.), Psiquiatría y anti psiquiatría (pp. 13-25). Buenos Aires, Argentina: Locus hypocampus. [Versión Adobe Reader]. Recuperado de https://colectivoantipsiquiatria.files.wordpress.com/2014/08/psiquiatria-y-antipsiquiatria-davidcooper-colectivoantipsiquiatria-wordpress-com.pdf.

Eidelsztein A. (02 de septiembre de 2015). La responsabilidad subjetiva. En Revista El Rey está desnudo 8(6), pp. 1-15. Recuperado de https://elreyestadesnudo.com.ar/wp-content/uploads/2015/09/La-responsabilidad-subjetiva.pdf.

Foucault, M. (2001/1953). Una conciencia política. En Perujo, F. (Trad.). El nacimiento de la clínica: una arqueología de la mirada médica (pp. 42-63). México: Siglo veintiuno editores.

Foucault, M. (2015/1964). Historia de la locura en la época clásica I. Buenos Aires, Argentina: Fondo de Cultura Económica.

Freud, S. (1972). El porvenir de la terapia psicoanalítica. En Ballesteros, L. (Trad.). Psicoanálisis aplicado y técnica psicoanalítica (Vol. XLVII, pp. 6672). España: Mandius ePub, Proyecto Scriptorium: Ex Libris.

Freud, S. (1991a/1916-1917). Conferencia 16: Psicoanálisis y psiquiatría. En J. Strachey (Ed.) y J.L. Etcheverry y L. Wolfson (Trads.). Obras completas (Vol. XVI, pp. 223-234). Buenos Aires, Argentina: Amorrortu. 
Freud, S. (1991b/1911-1913). Recordar, repetir y reelaborar (Nuevos consejos sobre la técnica del psicoanálisis, II). En J. Strachey (Ed.) y J.L. Etcheverry y L. Wolfson (Trads.). Obras completas (Vol. XII, pp. 145-157). Buenos Aires, Argentina: Amorrortu.

Freud, S. (1992a/1920-1922). Dos artículos de enciclopedia: Psicoanálisis y Teoría de la libido. En J. Strachey (Ed.) y J.L. Etcheverry y L. Wolfson (Trads.). Obras completas (Vol. XVIII, pp. 227-254). Buenos Aires, Argentina: Amorrortu.

Freud, S. (1992b/1920-1922). El psicoanálisis. En J. Strachey (Ed.) y J.L. Etcheverry y L. Wolfson (Trads.). Obras completas (Vol. XVIII, pp. 231-249). Buenos Aires, Argentina: Amorrortu.

Freud, S. (1992c/1900). La interpretación de los sueños. En J. Strachey (Ed.) y J.L. Etcheverry y L. Wolfson (Trads.). Obras completas (Vol. IV, pp. 1-16). Buenos Aires, Argentina: Amorrortu.

Freud, S. (1992d/1920-1922). La masa y la horda primordial. En J. Strachey (Ed.) y J.L. Etcheverry y L. Wolfson (Trads.). Obras completas, (Vol. XVIII, pp. 116-121). Buenos Aires, Argentina: Amorrortu.

Freud, S. (1992e/1901-1905). Sobre psicoterapia. En J. Strachey (Ed.) y J.L. Etcheverry y L. Wolfson (Trads.). Obras completas (Vol. VII, pp. 243-257). Buenos Aires, Argentina: Amorrortu.

Goffman, E. (2007). Sobre las características de las instituciones totales. En Oyuela, M. (Trad.). Internados. Ensayos sobre la situación social de los enfermos mentales (pp. 15-129). Buenos Aires, Argentina: Amorrortu.

Greiser, I. (2012a). El niño generalizado del mundo globalizado: la responsabilidad en la época actual. En Greiser, I. Delito y trasgresión (pp. 73-77). Argentina: Grama Ediciones.

Greiser, I. (2012b). El psicoanálisis en la época de la regulación. En Greiser, I. Psicoanálisis sin diván (pp. 43-64). Argentina: Paidós.

Lacan, J. (2001/1975). Introducción a los comentarios sobre los escritos técnicos de Freud. En Grancica, J. (Ed.). y Cevasco, R. \& Mira, V. (Trads.), Los escritos técnicos de Freud (pp. 19-35). Buenos Aires, Argentina: Editorial Paidós.

Lacan, J. (2003a/1966). Intervención sobre la transferencia. En Segovia, T. (Trad.). Escritos (Tomo 1. p. 204-215). México: Siglo XXI Editores.

Lacan, J. (2003b/1966). La ciencia y la verdad. En Segovia, T. (Trad.). Escritos (Tomo 2. pp. 834-856). México: Siglo XXI Editores.

Lacan, J. (2012/1967). Alocución sobre la psicosis del niño. En Esperanza G., Trabas, G., Tendlarz, S., Palomera, V., Álvarez, M., Delmont-Mauri, J., Sucre, J. \& Vicens, A. (Trads.). Otros escritos (pp. 381-391). Buenos Aires, Argentina: Editorial Paidós. 
Laurent, E. (2000). ¿Mental? En Laurent, E. Psicoanálisis y salud mental (pp. 135-138). Argentina: Editorial Tres Haches.

Miller, J. (2001). Psicoanálisis puro, psicoanálisis aplicado y psicoterapia. En Revista psicoanalítica, 32. Recuperado [con suscripción] de https:// www.freudiana.com/psicoanalisis-puro-psicoanalisis-aplicado-y-psicoterapia/.

Miller, J. (2010). Salud mental y orden público. España: Universidad de Granada, Escuela Lacaniana de Psicoanálisis Libros, Colección ELP/RBA.

Pérez, J. (2010). Acerca del concepto de salud mental. En Ruiz, L. (Ed.), El silencio de los sintomas: la salud mental (pp. 9-18). Medellín, Colombia: Nueva Escuela Lacaniana (NEL) de Medellín.

Quintero, J. (2017). El sujeto de la clínica. Cali, Colombia: Editorial Bonaventuriana.

Roudinesco, E. (2005). Diccionario de psicoanálisis. Buenos Aires: Paidós. 